\title{
On The Identification of Sonar Features
}

\author{
Simon LACROIX* \\ LAAS/CNRS \\ 7. Ave du Colonel Roche \\ 31077 Toulouse Cedex 4 FRANCE \\ Simon.Lacroix@laas.fr
}

\author{
Gregory DUDEK \\ Computer Science, McGill University \\ 3480 University Street \\ Montreal,Que, H3A 2A7, CANADA \\ Gregory.Dudek@cim.mcgill.ca
}

\begin{abstract}
In this paper, we are interested in inferring the sources of various types of sonar features typically observed by a mobile robot. After a brief discussion of terrestrial sonar sensing, we develop a set of operators that associates arc-shaped features extracted from sonar scans with real world primitives. Our classification scheme is probabilistic and is based on empirical data: the confidence of the association hypotheses produced by the operators is evaluated statistically. Some of our experimental results suggest that methods based on models of perfect sonar sensors may not be completely consistent with observed data. The management and merging of a collection of hypotheses concerning various sonar features allows the system to produce a coherent and mutually-compatible set of inferences for the entire observed environment.
\end{abstract}

\section{Introduction}

Sonar data is frequently used for map construction and position estimation. The most common models for sonar data involve representing a sonar echo as a data point at a distance given by the time of flight along a line normal to the transducer. Collections of these data points are then used to build a grid-based environment model [1, 2], or modeled using linear geometric primitives. Under the best conditions, some of these geometric models match physical structures in the environment while others are the results of echos that have bounced on several surfaces. Traditional approaches to modeling sonar data involve generating geometric primitives from either a single position, or by combining data from multiple positions to minimize either a squared error measure between the data and the models, or via an Extended Kalman Filter [3, 4, 5].

Circular geometric models typify the pattern of responses obtained by taking a series of observations at different orientations from a fixed position; these models are known as "regions of constant depth (RCDs)" [6]. By a careful comparison of the size of circular models, one can derive an analytic expression

*This work was carried out at the Centre for Intelligent Machines, McGill University, where the first author held a one-year fellowship sponsored by INRIA. The support of the Canadian National Sciences and Engineering Research Council, via a grant to the second author, is also gratefully acknowledged. that allows for the discrimination of walls and corners [7]; in principle, this allows some of the original environmental structure of be inferred. However, such methods depend on important assumptions regarding the makeup of the environment. To our knowledge, they have not been extensively evaluated in practice on functioning "standard" robots.

In this paper, we focus only on primitive identification, and assume the robot's absolute position is always known. We show that some of the distinctions between different types of sonar features based on "well-behaved" environments, with very consistent reflectance properties, appear to be rather unreliable in practice. In contrast, we show that various types of feature classification are possible in a probabilistic context. The next section recalls well known properties of terrestrial sonar sensors, presents the sonar features (RCDs) used to model the environment, and describes the model we aim to build. Section 3 introduces the basic technique we propose to generate primitives/RCDs associations, based on a Bayesian classification procedure. Section 4 presents how RCDs extracted from data sets acquired from various viewpoints are matched, and how the matches can lead to a primitive identification. Section 5 explains how association hypotheses are managed to produce a coherent model.

\section{Sonar features and world primitives}

\subsection{Sonar sensors characteristics}

A standard model for sonar sensing is to represent the sonar sound wave as a ray that is emitted normal to the transducer surface, and whose echo returns along the same path (we will refer to this as the ray model). Unfortunately, this model would be optimistic even for a laser range sensor. However, the understanding of the physics of sound waves has permitted faithful modeling of sonar sensing devices $[8,9,10]$. We briefly review here the properties of this model, as they play a critical role in the development of our technique.

Beam width: A nominal range estimate can be obtained directly from the delay time in the reception of an acoustic echo. The ultrasonic wave emitted by a 
sonar sensor transmitter is contained in a lobe ${ }^{1}$ with a $2 \alpha$ angular width (nominally $46^{\circ}$ for the Polaroid transducers we use). In many cases, it is the side of this beam that reflects off a surface in the environment (Figure 1): as a consequence, the returned ranges are often much smaller than one would expect with the simplified ray model. This phenomenon also occur when perceiving edges, and actually with many objects present in an indoor environment.
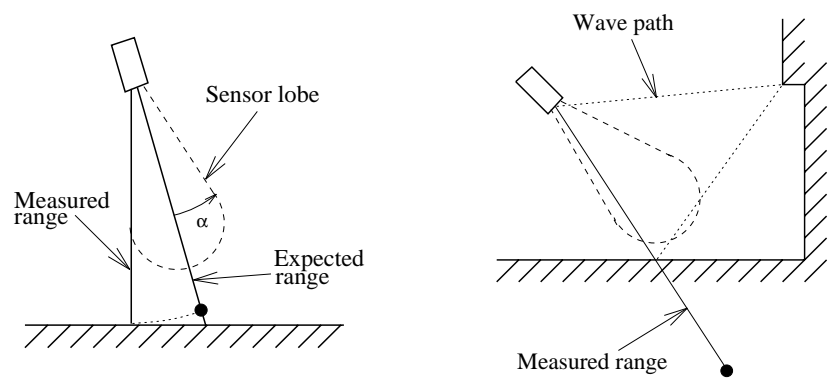

Figure 1: Consequences of the beam width (left) and the surface specularity (right) on the measured ranges

Surface specularity: Most of the surfaces that make up typical indoor environments act as specular reflecting surfaces at ultrasonic frequencies. When the sonar wave hits such a surface, it is reflected such that the angle of reflection is roughly equal to the angle of incidence. As a consequence, the sonar beam is reflected away from the transducer for surfaces that are not almost facing it. In this case, either no echo is detected by the receiver, or an echo corresponding to multiple reflections with other objects is detected [11] (Figure 1). The latter case is known as the "multiple reflection" phenomenon, and produces totally erroneous range measurements. Depending on the number $n$ of object encountered during the beam propagation, the sonar echo is said to be a $n^{t h}$ order echo [6].

Diffraction: When the sonar beam hits an edge, it is diffracted: the resulting echo wave spreads cylindrically from the corner. The signal received by the sensor has then a much lower amplitude than the echo produced by a wall since the energy is dissipated widely [8]. The maximal distance from which an edge can be perceived is then smaller than for walls.

These properties have an important consequence: any interpretation of sonar data based on the ray model exhibits many artifacts, such as apparent failures to detect nearby obstacles (due to specularity or diffraction), or spurious objects ( $n^{\text {th }}$ order echos). As a consequence, sonar sensors as a basis for environment mapping have been regarded with suspicion within the robotics community, especially since laser range sensors have become readily available.

\footnotetext{
${ }^{1}$ Sonar sensors also emit waves in secondary side lobes, but the energy is much smaller than in the central lobe: we actually never observed any range measure outgoing from one of these lobes during our experimentations.
}

\subsection{Sonar scans and RCDs}

We interpret panoramic sonar scans acquired with a ring of 16 Polaroid sensors on our Nomad 200 robot: a set of data taken after several equal rotations of the robot produces a sonar scan. Figure 2 presents such a scan ${ }^{2}$, superimposed on a map of the environment. Note that several clusters of points form circular arcs (or RCDs) corresponding either to walls (clusters 1,3 , and 7), corners (clusters 2, 9, and 6), cylinders (cluster 8 ), edges (cluster 10) or multiple reflections (clusters 4, 5 , and 15). The arcs on top of the figure are produced by unmapped chairs and tables.

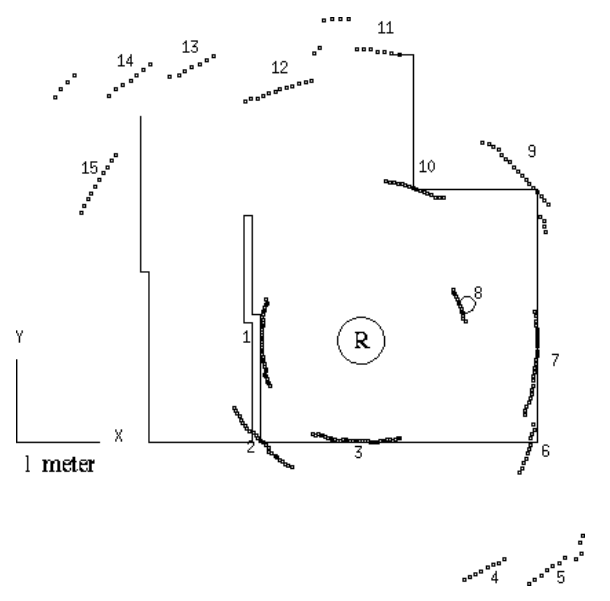

Figure 2: A 256 points sonar scan

The way RCDs are produced by corner, wall and edge primitives is clearly a consequence of the sonar properties (Figure 3 ):

- The echo reflected by a wall reaches the sensor receiver only if the sonar is directed toward the wall with an angle comprised in $[-\alpha, \alpha]$. At higher angles of view, the side of the sonar lobe hits the wall at such an angle that the wave is reflected away. Theoretically, when perceiving a purely specular wall, the width $\Delta \theta$ of the RCD is then twice the sonar lobe width.

- In the case of a corner, the received echos are the one that are reflected twice at the corner angle. Similar considerations as for the RCDs produced by walls lead to the conclusion that the corner RCDs width is theoretically also twice the lobe width.

- The echo returned by edges is due to diffraction. Using wave propagation theory, R. Kuc showed that the width of the corresponding arc should be smaller than for walls and corners [12].

Our technique is based on statistically-observed properties of individual RCDs in the sonar scan. RCDs are extracted by the application of a threshold on the distance between successive range data points. An RCD defined by its distance to the sensor $r$ and two bounding angles $\left(\theta_{\min }, \theta_{\max }\right)$. Associated parameters are the angular width $\Delta \theta$, and an absolute orientation $\theta=\left(\theta_{\min }+\theta_{\max }\right) / 2$. There are, of course, uncertainties in the values of these parameters: a stability

\footnotetext{
${ }^{2}$ Throughout the paper, we will deal with 256 points sonar scans.
} 

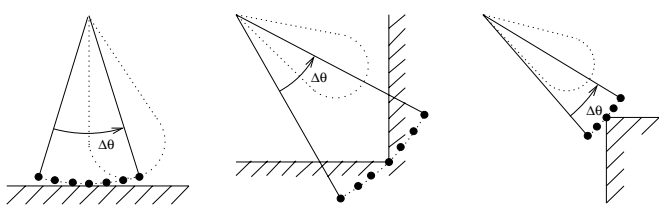

Figure 3: RCDs generated by a wall, a corner and an edge. The leftmost sonar lobe of each $R C D$ is shown.

analysis over several scans acquired from the same position shows a standard deviation in the feature's orientation of roughly 3 degrees. The origin of these deviations is that points at the edges of an RCD typically have amplitudes near the thresholds of detectability for the transducer (that is why the cluster ends at these points). Thus these terminal points are very unstable: small amounts of noise (either acoustic or electrical) lead to the appearance or disappearance of a data point ${ }^{3}$. We could not precisely determine the uncertainty in the distance to a RCD as it is below the accuracy threshold for our transducers: the value returned by the sensors is coded with insufficient precision ( 8 bits encoding ranges up to 8.0 meters) ${ }^{4}$. However, the returned range appears quite stable: when a feature is detected, it is consistently detected.
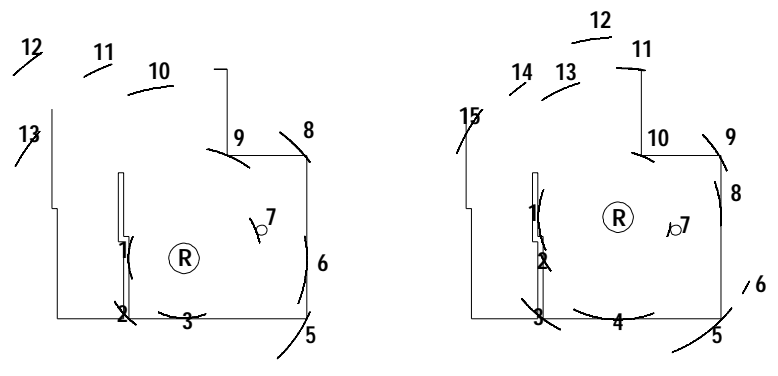

Figure 4: Two "RCD scans" extracted from sonar scans acquired in the environment mapped in Figure 2.

\subsection{World primitives}

We model the environment as a collection of primitives classified into the following types:

- Walls $W$ are defined by a supporting line defined by $(\phi, R)$ in a global frame and two endpoints.

- Corners $C$ correspond to a right-angled intersection of two walls, or to a "break" in a wall (see Figure 5). They are defined as "oriented" points.

- Edges E correspond to the ending of a wall or to a normal intersection of two walls; they are also defined as "oriented" points.

- Cylinders $C y$ are defined by the position $P(x, y)$ of their center, and a radius $r$.

There are numerous objects that generate RCDs and that do not correspond to any of these primitives (chairs, shelves... often composed of numerous edges

\footnotetext{
${ }^{3}$ We therefore chose to keep only RCDs that contain more than 5 points for subsequent interpretation purpose

${ }^{4}$ New hardware has alleviated this problem but the revised data was not available at this time.
}

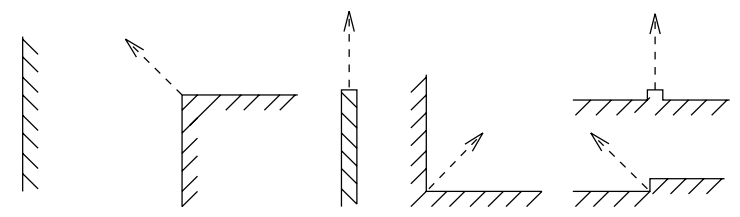

Wall

Edges

Corners

Figure 5: The primitives of our world model. The dashed arrows represent the "directions" of corners and edges

and cylinders). Thus, we define an additional primitive type that represents these cluttered areas $(\mathrm{Cl})$, to describe anything not accounted for by one of the prior primitives. Finally, when an RCD corresponds to a third or higher order of reflection, it will be identified as a multi-bounce $\operatorname{RCD}(M u)$.

Any observed RCD corresponds then to one of the primitives in the set $\mathcal{P}=\{W, C, E, C l, M u\}$. An hypothesis $H\left(F_{j}, P_{i}\right)$, where $F_{j}$ is an RCD and $P_{i} \in \mathcal{P}$, is a correspondence between one primitive and one feature (one feature can be associated with several primitives, and one primitive can be associated with several features). Each hypothesis has an associated probability $P_{H\left(P_{i}, F_{j}\right)}$ of existence.

\section{Local primitive extraction}

Using existing models of sonar sensors, it is difficult in practice to establish the different sources of RCDs. This is one of the reason why sonar arrays have been introduced to map the environment $[13,14,15]$. However, we establish in this section that statistical analyses of the attributes of a single RCD scan can be used to determine its causative source, sometimes with high confidence.

\subsection{RCDs classification}

According to [8], only edges can be differentiated from corners or planes using the angular width of an RCD (in an occlusion-free environment). But an analysis of empirical sonar data scans reveals that the angular width can lead to a better differentiation. To clearly establish this, we manually classified and examined a set of 750 RCDs extracted from sonar scans, taken from various positions in our lab. Figure 6 shows the probability density for the width of an RCD for various types of feature $\left(P\left(\Delta \theta \mid P_{i}\right) / P(\Delta \theta)\right)$. These data lead to several interesting observations:

- The width of RCDs corresponding to walls have a peak probability around the value $\Delta \theta=45^{\circ}$ (twice the sonar lobe width; smaller widths correspond to partially occluded walls).

- Corner RCDs also have a peak at roughly $15^{\circ}$, and seldom reaches the wall's peak value, which confirms that $\Delta \theta$ is a quite discriminant attribute to distinguish them from wall RCDs [7].

- Edges also have a peak around a much smaller value $\left(15^{\circ}\right)$ than walls, which confirm the theoretical models validity. However, these models predict that edge RCD widths is a function of the distance from which they are observed [7]: we have not observed this dependence on our edge prototypes: this may relate to 

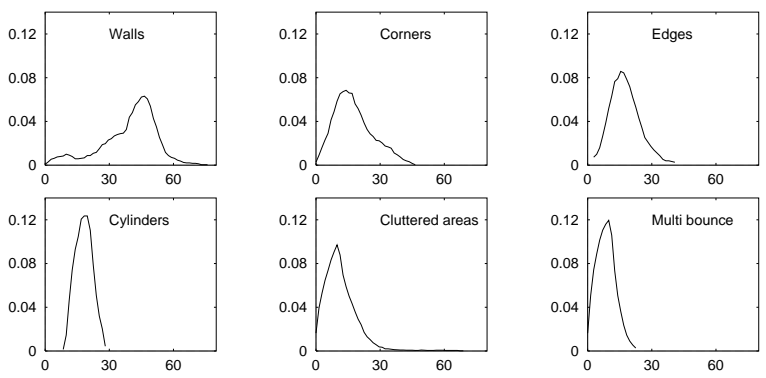

Figure 6: Probability density functions of $R C D$ widths

the fact that our experimental data was collected in an environment several meters wide.

- As for multi-bounce RCDs, their width never exceeds $25^{\circ}$ and has a peak around $10^{\circ}$.

The data for cylindrical targets is not conclusive and is included for illustrative purposes only: only a single $7 \mathrm{~cm}$ diameter cylinder was present in our test data (it produced data much like an edge).

If we suppose that walls are almost all oriented along two orthogonal directions (a reasonable heuristic in indoor environments), the histogram of their orientations is composed of two peaks, separated by a distance of 90 degrees. Corners are then also oriented along two normal directions, that make a $45^{\circ}$ angle relative to the associated walls. This hypothesis obviously has implications for the corner and walls RCDs absolute orientation, but also on the multi-bounce RCDs, which appear when perceiving a wall with an angle of incidence greater than the sonar lobe width. Figure 7 shows the probability density functions $P\left(\theta \mid P_{i}\right)$ of the angular orientation for our 750 prototypes, for RCDs corresponding to walls, corners and multi-bounces ${ }^{5}$. Note that using $\theta$ as an attribute to identify RCDs requires the knowledge of the robot orientation with respect to an absolute coordinate frame, which we assume provided by a localization algorithm.

Using these a priori probability density functions, we can now estimate the probability of hypotheses $H\left(F_{j}, P_{i}\right)$ using the Bayes rule:

$$
P_{H\left(P_{i}, F_{j}\right)}=P\left(P_{i} \mid \Delta \theta_{j}, \theta_{j}\right)=\frac{P\left(\Delta \theta_{j}, \theta_{j} \mid P_{i}\right) P\left(P_{i}\right)}{P\left(\Delta \theta_{j}, \theta_{j}\right)},
$$

where $P\left(P_{i}\right)$ is the a priori probability of the primitive $P_{i}$ to be present in the environment, and

$$
P\left(\Delta \theta_{j}, \theta_{j}\right)=\sum_{P_{i} \in \mathcal{P}} P\left(\Delta \theta_{j}, \theta_{j} \mid P_{i}\right) P\left(P_{i}\right)
$$

is the probability for an RCD has width $\Delta \theta_{i}$ and an orientation $\theta$. A priori probability density functions are determined with a non-parametric nearest neighbors technique, and in the absence of other knowledge, we can assume that primitives are uniformly distributed in the environment.

\footnotetext{
${ }^{5}$ The other primitives (cylinders, edges and cluttered areas) orientation repartitions are not plotted: they are of course uniform.
}
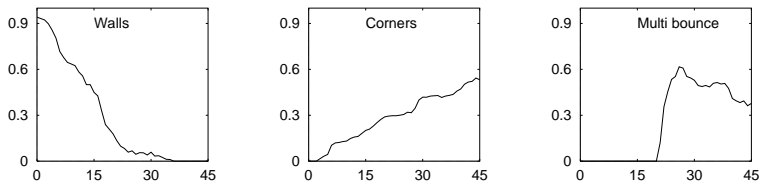

Figure 7: probability density functions of $R C D$ orientation. Due to symmetry, the functions are plotted between 0 and $45^{\circ}$ )

Table 1 presents the probability of the various hypotheses using the width and orientation attributes for several of the RCDs from Figure 2. This illustrates that some RCDs can be quite faithfully identified directly from this data: in particular, unoccluded walls can be detected due to the large width of their corresponding RCD.

\begin{tabular}{|c|c|c|c|c|c|}
\hline$R C D$ & $1(\mathrm{~W})$ & $2(\mathrm{C})$ & $6(\mathrm{C})$ & $10(\mathrm{E})$ & $15(\mathrm{Mu})$ \\
\hline$\Delta \theta$ & 49.2 & 33.8 & 14.1 & 21.1 & 12.1 \\
\hline$\theta$ & 1.6 & 44.5 & 12.1 & 25.5 & 14.2 \\
\hline$P(W)$ & 1.00 & 0.00 & 0.00 & 0.06 & 0.01 \\
\hline$P(C)$ & 0.00 & 0.97 & 0.58 & 0.35 & 0.49 \\
\hline$P(E)$ & 0.00 & 0.02 & 0.05 & 0.21 & 0.06 \\
\hline$P(C y)$ & 0.00 & 0.00 & 0.07 & 0.31 & 0.07 \\
\hline$P(C l)$ & 0.00 & 0.01 & 0.03 & 0.07 & 0.04 \\
\hline$P(M u)$ & 0.00 & 0.00 & 0.26 & 0.00 & 0.34 \\
\hline
\end{tabular}

Table 1: Association hypotheses probabilities for several RCDs of figure 2, using the RCDs width and absolute orientation

\subsection{Finding corners}

One of our objectives is to combine hypotheses across features in a relaxation-like frame to produce a more consistent and robust interpretation. The following procedure illustrates the first step in this direction.

We can exploit the hypotheses that corners are produced by a pair of mutually orthogonal walls to define an additional inference process for corner primitives. Indeed, the attributes of a triplet $\left\{F_{1}, F_{2}, F_{3}\right\}$ of RCDs produced by 2 orthogonal walls and the associated corner (such as the triplets $\{1,2,3\}$ and $\{3,6,7\}$ in Figure 2 ) should satisfy the following relations :

$$
\begin{gathered}
\theta_{F_{3}}-\theta_{F_{1}}=\pi / 2 \\
\Omega\left(F_{1}, F_{2}, F_{3}\right)=\sqrt{r_{F_{1}}^{2}+r_{F_{3}}^{2}}-r_{F_{2}}=0
\end{gathered}
$$

where $\left\{F_{1}, F_{3}, F_{2}\right\} \equiv\{W, C, W\}$.

We classified and analyzed all of the RCD triplets whose corresponding primitives are contained in a $90^{\circ}$ angular sector (i.e. the ones that satisfy $\left\|\theta_{F_{3}}-\theta_{F_{1}}\right\| \leq$ $\pi / 2+\alpha)$ in our sonar scans, and discovered that the relation (1) is actually of little utility for detecting corners. This is due to errors in the estimates of the orientation of the RCD, to occlusions that produces wall RCDs with small width and biased orientation, and to the fact that numerous pairs of arbitrary RCDs can satisfy it, without being associated with a pair of two orthogonal walls. The second relation $\Omega$, however, provides a powerful discriminator (Figure 8): most of the triplets that are not due to actual corners produce 
a large value of the estimator (2), whereas this value is always below $0.15 \mathrm{~m}$ for triplets associated with actual corners.
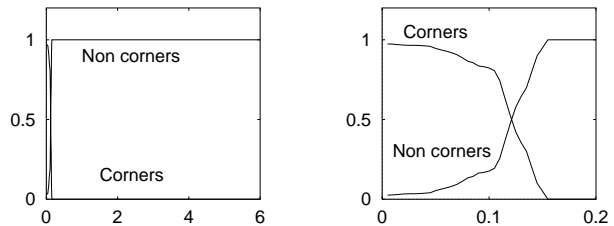

Figure 8: Relative pdf's as a function of the estimator $\Omega$ (Equation 2) for corners and non-corners triplets. Entire range (left) and zoomed-in relevant subregion (right)

Table 2 presents the triplets extracted from the scan shown in Figure 2 to which a non-null probability of representing a wall-corner-wall triplet has been computed using to the probabilities produced by the second estimator value. The 2 corners present in the environment are detected with a high associated probability, and only three supplementary triplets (out of 170 triplets that satisfy $\left\|\theta_{F_{3}}-\theta_{F_{1}}\right\| \leq \pi / 2+\alpha$ in the scan) have a non null probability to represents corners.

\begin{tabular}{|c|c|c||c|c|c|}
\hline Triplet & $1,2,3$ & $3,6,7$ & $7,9,10$ & $8,11,12$ & $13,14,1$ \\
\hline$P_{\{W, C, W\}}$ & 0.97 & 0.92 & 0.82 & 0.91 & 0.30 \\
\hline
\end{tabular}

Table 2: The RCD triplets from the $R C D$ scan of Figure 2 for which the estimator (2) returns a non-null probability to match a $\{W, C, W\}$ primitive triplet. The three rightmost triplets are false detections.

\section{Matching RCDs}

Observing changes in RCD characteristics as a function of changes in robot position can provide important information for classification. Indeed, it is easy to understand how two RCDs corresponding to the same primitive perceived from two different positions are related: a pair of corner or edge RCDs should intersect at their center, and a pair of RCDs associated with a single wall should be "parallel". It is difficult to predict how multi bounce RCDs will relate without knowing the surfaces which generated them. In general, they are highly unstable.

Prior work can considered the evolution of sonar data with robot motion. This idea was introduced [7] to differentiate walls from corners, and they play a central role in the algorithms developed in [6] and [16]. Similar considerations have been applied to build a qualitative description of the environment [17]. We consider here how RCDs extracted from two different positions are matched, and how numerical relations on their attributes can be analyzed to identify them.

\subsection{Determining matches}

Once two RCD scans have been acquired from two different positions, one must find the pairs (matches) of RCDs that actually correspond to the same primitive. This is accomplished by checking the following constraints :

$$
\begin{gathered}
\left\|r_{1}-r_{2}\right\| \leq\left\|P_{1} P_{2}\right\| \\
\left\|C_{1} C_{2}\right\| \leq\left\|P_{1} P_{2}\right\| \\
\sin \left(\left\|\theta_{1}-\theta_{2}\right\|\right) \leq\left\|P_{1} P_{2}\right\| / r_{1} \\
\Delta \theta_{1}=\Delta \theta_{2}
\end{gathered}
$$

These constraints are derived from geometric relations easily established on the illustrations shown in Figure 9. Note that they hold only for small robot motions (constraint (5), in particular, is undefined when $\left.\left\|P_{1} P_{2}\right\| \geq r_{1}\right)$, and that they are satisfied for RCD pairs produced by walls and edges, but also by corners and small cylindrical primitives whose matchings behave much like edges.
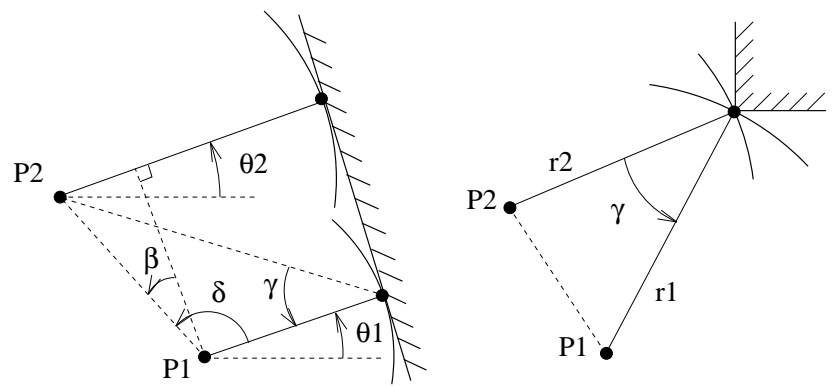

Figure 9: Objects observed from two different positions and their associated $R C D$ s, for a wall (left) and an edge (right)

The matching procedure then entails simply checking these constraints on all the possible RCD pairs that can be defined in two RCD scans. In practice, the thresholds are slightly relaxed to cope with the uncertainties on the RCD attributes and the robot position. False matches can occur, but we have discovered that when matches are unequivocally established (i.e. when an RCD from the first scan can be matched with only one RCD from the second scan), they are consistent. This matching procedure works very well for less than one meter translations.

\subsection{Matches identification}

Matches can be used to differentiate wall RCDs from corner and edge RCDs. As can be seen on Figure 9, the RCDs orientation remains stable for wall primitives, whereas it changes for other primitives, and there are

\begin{tabular}{|c|c|c|}
\hline & Walls & Others \\
\hline||$\theta_{2}-\theta_{1} \mid$ & 0 & $\geq 0$ \\
\hline$r_{2}-r_{1}+\sin (\beta)$ & 0 & $\leq 0$ \\
\hline
\end{tabular}
formulas that relates two wall RCDs distance with the robot motion that are not satisfied for other primitives matches. We retained the following estimators ${ }^{6}$ :

If the robot moves on a line normal to an RCD, the new observations will assure that the estimators are identically equal to zero in all cases. The estimators become more discriminative as the direction of robot motion diverges from the orientation of the first RCD,

\footnotetext{
${ }^{6}$ The predicted result that two corner RCDs intersect at their center [7] was rarely observed with our empirical data
} 
and as the distance moved increases. These is illustrated by the angle $\gamma$ in Figure 9:

$$
\tan \gamma=\frac{\left\|P_{1} P_{2}\right\| \sin \delta}{r_{1}-\left\|P_{1} P_{2}\right\| \cos \delta} .
$$

Figure 10 shows the values of the two estimators as a function of $\gamma$ for a set of prototyped matches. One can see that matches can not be differentiated for a value of $\gamma$ below $10^{\circ}$, and that the distribution of estimator values as a function of $\gamma$ can be confidently partitioned into regions for large $\gamma$. The interpretations of the matches between RCDs of the two scans shown in Figure 4 are presented in Table 3.
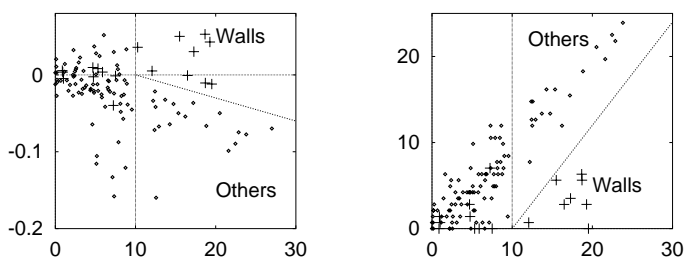

Figure 10: Value of the angular variation (left) and of the second estimator (right) as a function of $\gamma$ for a set of prototyped matches (t signs are walls prototypes, and dots are the other primitives prototypes).

\begin{tabular}{|c|c|}
\hline RCDs matched & Decision \\
\hline $1_{1}$ and $1_{2}$ & Wall \\
\hline $2_{1}$ and $3_{2}$ & No decision \\
\hline $3_{1}$ and $4_{2}$ & Wall \\
\hline $5_{1}$ and $5_{2}$ & Not a wall \\
\hline $7_{1}$ and $7_{2}$ & Not a wall \\
\hline $8_{1}$ and $9_{2}$ & No decision \\
\hline $9_{1}$ and $10_{2}$ & No decision \\
\hline $10_{1}$ and $13_{2}$ & Not a wall \\
\hline
\end{tabular}

Table 3: Identification of the matches from the scans of Fig. 4

\section{Hypotheses management}

Up to now, we defined three operators that generate associations hypotheses. Below, we outline a simple approach to integrate the informations provided by these estimators to produce a coherent world model.

\subsection{Merging local informations}

The corner extraction operator estimates the probability $P_{\left(H\left\{\left\{F_{1}, F_{2}, F_{2}\right\},\{W, C, W\}\right)\right.}$ that the triplet of RCDs $\left\{F_{1}, F_{2}, F_{3}\right\}$ corresponds to the primitive triplet $\{\mathrm{W}, \mathrm{C}, \mathrm{W}\}$. An estimation of the same probability is also provided by the RCDs classification procedure, by multiplying the individual identification probabilities: $P_{\left(H\left(\left\{F_{1}, F_{2}, F_{2}\right\},\{W, C, W\}\right)\right.}=P_{H\left(F_{1}, W\right)} P_{H\left(F_{2}, C\right)} P_{H\left(F_{3}, W\right)}$.

These two estimations can be fused thanks to the Bayes rule. This combination is of a very good help to discard the false detection of the corner extractor: one can see on the table 4 that the three triplets of the scan from Figure 2 that do not correspond to a $\{\mathrm{W}, \mathrm{C}, \mathrm{W}\}$ triplet have a very low score after the combination.

\begin{tabular}{|c|c|c||c|c|c|}
\hline Triplet & $1,2,3$ & $3,6,7$ & $7,9,10$ & $8,11,12$ & $13,14,1$ \\
\hline$P_{\{W, C, W\}}$ & 0.97 & 0.92 & 0.82 & 0.91 & 0.30 \\
\hline$P_{W} P_{C} P_{W}$ & 0.97 & 0.51 & 0.04 & 0.00 & 0.00 \\
\hline Combination & 0.99 & 0.92 & 0.16 & 0.00 & 0.00 \\
\hline
\end{tabular}

Table 4: Results of the introduction of the informations provided by the individual RCDs classification on the corners detected in the RCD scan of Figure 2 : the false detections are easily discarded

\subsection{Constraint propagation}

We can introduce two realistic "primitive completion constraints" that links the primitives together (they can be considered as a part of the primitives definition):

- Each wall is ended by an edge, a corner or a cluttered area.

- Each edge or corner is supported by two walls that are normal or parallel.

Walls, corners and edges primitives are said to be completely detected when their ending or supporting primitives are identified.

Let's consider that the RCD triplet $\{2,4,6\}$ of Figure 11 has been identified as a $\{W, C, W\}$ primitive triplet (i.e. RCD 4 is a completely detected corner). From this hypothesis, we can derive new hypotheses:

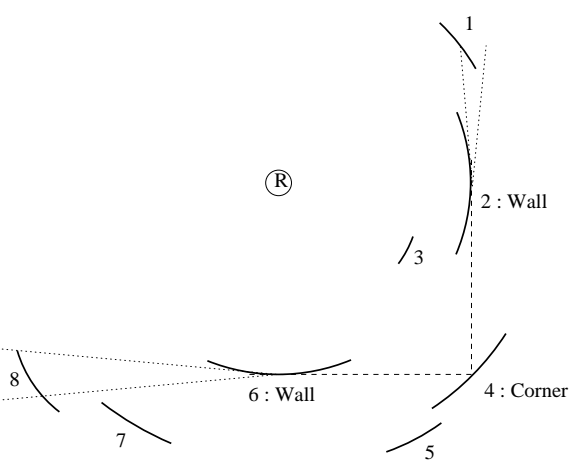

Figure 11: A set of RCDs in which a corner has been completely detected

- RCD 5 must be a multi bounce RCD. Indeed, it is located behind the line supported by RCDs 4 and 6 , which corresponds to a wall ;

- RCD 3 must be a primitive that can not be completed (a cylinder or a cluttered area): it is located in front of the line supported by RCDs 2 and 4 (a wall), and can therefore not be a multi bounce RCD ;

We can also seek possible completions for uncompleted hypotheses (walls 2 and 6 in this example). For instance, RCD 8 may correspond the right end of the wall supported by RCD 6 (it intersects an angular sector that bounds the orientation uncertainty on RCD 2 , the dotted line in the figure). But in this case, RCD 7 would be a multi bounce RCD, which is impossible because of its large width (see section 3.1): RCD 8 can not correspond to the right ending of wall 6 . And since there are no other RCDs between 6 and 8 , the right 
ending of wall 6 must be an edge, located somewhere between RCD 6 and RCD 7 .

This example illustrate the kinds of inference that can be performed on the basis of confident hypotheses. The difficulty here relies on the hypotheses management and probability evaluation: a good formalization of the relations between hypotheses (dependencies, compatibilities) is required, and a relaxation-like formalism should be applied to update the likelihood of the hypotheses. Various contributions within an object recognition framework could be adapted to such a problem.

\subsection{Results}

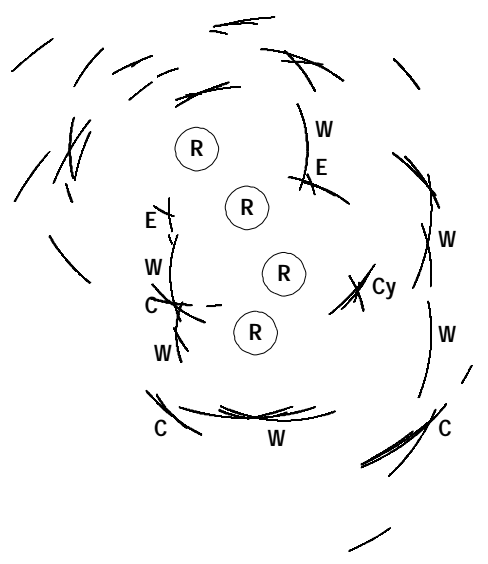

Figure 12: Accumulation of RCDs observed after 4 data scans. Labeled primitives are those with an hypothesis with a likelihood greater than 0.9

In preliminary trials, we have considered simple illustrative heuristics to combine hypotheses. Walls detected via matching (Sec 4.2) and corners detected as above have their association probability set to 1 . As for the other results of the matching process (i.e. those features with the "Not a wall" labeling), they are "projected" on the RCD's individual classification: the wall probability for the two RCDs is set to zero, and the remaining partial probabilities are normalized. Finally, the partial probabilities of the matched RCDs are combined using Bayes rule.

Figure 12 presents an environment model built from 4 sonar acquisitions, in which only the hypotheses whose likelihood value is greater than 0.9 are kept. Despite the simplicity of the rules, the labels that are produced appear to be be completely correct.

\section{Discussion}

We have shown that it is possible infer the environmental source of sonar features. Using statistical geometric properties derived from a large data set, we have developed various processes that are able to assign likelihoods to associations between sonar features and explanatory hypotheses. While prior work on theoretical and idealized modeling is highly relevant, it appears to suffer from certain limitations in practice. Indeed, such models are not directly applicable to sonar data acquired in a realistic application context.

The way the association hypotheses generated by these processes are combined and managed is for the moment essentially heuristic: we are currently tackling this problem within a more formal framework. Future work will focus on the process of active modeling, which consists in seeking the relevant data in order to both instantiate new hypotheses, to delete existing ones, or to update the certainty of existing hypotheses. The behavior of our primitive detection processes can be statistically modeled, and the utility of a point of view can therefore be determined.

\section{References}

[1] H. P. Moravec and A. Elfes. High resolution maps from wide angle sonar. In IEEE ICRA 1995

[2] J. H. Lim and D. W. Cho. Physically based sensor modeling for a sonar map in specular environment. In IEEE ICRA 1993

[3] M. Drumheller. Mobile robot localization using sonar. IEEE Transactions on and Machine Intelligence, 9(2):325-332, March 1987.

[4] J. L. Crowley. World modeling and position estimation for a mobile robot using ultrasonic ranging. In IEEE ICRA 1989

[5] P. MacKenzie and G. Dudek. Precise positioning using model-based :aps. In IEEE ICRA 1994

[6] J. J. Leonard, H. F. Durrant-Whyte, and I. J. Cox. Dynamic map building for an autonomous mobile robot. The International Journal of Robotics Research, 11(4):286-298, August 1992.

[7] O. Bozma and R. Kuc. Building a sonar map in a specular environment using a single mobile sensor. IEEE Transactions on Pattern Analysis and Machine Intelligence, 13(12):1260-1269, December 1991.

[8] R. Kuc and M. W. Siegel. Physically based simulation model for acoustic sensor robot navigation. IEEE Transactions on Pattern Analysis and Machine Intelligence, 9(6):766-778, 1987.

[9] G. Dudek, M. Jenkin, E. Milios, and D. Wilkes. Reflections on modeling a sonar range sensor. Technical Report CIM-92-9, McGill Centre for Intelligent Machines, 1992.

[10] J. J. Leonard and H. F. Durrant-Whyte. Directed Sonar Sensing for Mobile Robot Navigation. Kluwer Academic Publishers, 1992.

[11] P. J. Mc Kerrow and S. M. Zhu. Modelling multiple reflcetion paths in ultrasonic sensing. In IEEE IROS 1996

[12] R. Kuc and B. Barshan. Navigating vehicles through an unstructured environment with sonar. In IEEE ICRA 1989

[13] B. Barchan and R. Kuc. Differentiating sonar reflections from corners and planes by employing an intelligent sensor. IEEE Transactions on Pattern Analysis and Machine Intelligence, 12(6):560-569, June 1990.

[14] L. Kleeman and R. Kuc. Mobile robot sonar for target localization and classification. The International Journal of Robotics Research, 14(4):295-318, Aug 1995. 
[15] A. Stevens, M. Stevans, and H.F. Durrant-Whyte. Oxnav : Reliable autonomous navigation. In IEEE ICRA 1995

[16] I. J. Cox and J. J. Leonard. Modeling a dyanmic environment using a bayesian multiple hypothesis approach. Artificial Intelligence, 66:311-344, 1994.

[17] S. Reece and H. Durrant-Whyte. A qualitative approach to sensor data fusion for mobile robot navigation. In IJCAI 1995 